\title{
Can Development Aid Change Attitudes Toward Refugees? Experimental Evidence from Urban Microentrepreneurs in Uganda: Pre-Analysis Plan*
}

\author{
Travis Baseler ${ }^{\dagger} \quad$ Thomas Ginn ${ }^{\ddagger} \quad$ Helidah Ogude ${ }^{\S}$ Olivia Woldemikael ${ }^{\llbracket}$
}

October 20, 2020

\begin{abstract}
How should assistance for refugees be allocated to aid refugees and gain the support of host communities? Host populations often believe they are negatively affected by refugees, yet the scope for assistance to facilitate positive relations and mitigate tension is largely unknown. We conduct a randomized controlled trial to investigate three programs for Ugandan microentrepreneurs that we saliently link to the refugee presence and Uganda's existing aid-sharing policy: canvassing, canvassing accompanied by a cash grant, and mentorship by an experienced refugee. We measure social outcomes, including support for hosting additional refugees and allowing them to work, and economic outcomes, such as business survival and profits, to test whether effective development aid associated with refugees can induce support for inclusive hosting.
\end{abstract}

\section{Overview of project}

Host countries often restrict refugees' access to the labor market because they are afraid inclusion would crowd out citizens. Even in many protracted situations, international donors can only provide humanitarian assistance like food aid that keeps refugees alive but has little expected long-run returns for refugees and host communities alike. We aim to study whether reallocating some assistance to the host community - especially assistance that is tied to refugees' access to work - could shift the political economy of hosting refugees from humanitarian aid and restrictive policy towards an equilibrium of shared development aid and inclusive policy. Policymakers are increasingly including host communities in assistance, but whether this shift can impact host citizens' policy preferences is an open question.

Uganda is perhaps the leading example of a positive arrangement between a host government and the humanitarian community. It is the largest host country in Africa yet allows refugees to work

${ }^{*}$ We referred to Benhassine et al. $(2015)$ as a template for drafting this plan.

${ }^{\dagger}$ University of Rochester, travis.baseler@rochester.edu

${ }^{\ddagger}$ Center for Global Development, tginn@cgdev.org

§The World Bank, hogude@worldbank.org

๑ Harvard University, woldemikael@g.harvard.edu 
and move freely. Refugees can locate in settlements and receive food assistance or, since 2006, live and start businesses nationwide. In return, $30 \%$ of aid to refugees must support Ugandans and often goes to health and education programs. In many ways, it is the model other hosting countries, most notably Ethiopia, are moving towards and learning from. Still, despite over 100,000 refugees living in Kampala, little assistance extends to urban areas, and most citizens are unaware that assistance for refugees is shared with Ugandans. We study micro-entrepreneurs - a large demographic of Ugandan hosts, male and female - who may be in competition with refugee entrepreneurs and are therefore important to target for social cohesion interventions.

We investigate three programs that saliently link the refugee presence and Uganda's existing aidsharing policy: canvassing, canvassing accompanied by a cash grant, and mentorship by an experienced refugee. Our goal is to emphasize complementarities between refugees' presence-both refugees themselves and the international assistance that comes to host communities from hosting refugees - and Ugandan hosts. The canvassing treatment highlights the complementarity that exists through the aid-sharing policy but not widely known. The canvassing with grants and the refugee mentorship treatments potentially induce new complementarities that directly benefit the business owners in our sample. We use two additional treatment arms to isolate mechanisms. First, we distribute cash grants with no accompanying information about refugees to measure whether the hypothesized increase in profits is sufficient to change attitudes. Second, we also organize mentorship by a Ugandan mentor to discuss the effect of the mentors' refugee status. Our main outcomes are both social outcomes, including support for hosting additional refugees and allowing them to work, and economic outcomes, such as business survival and profits, to test whether effective development aid associated with refugees can induce support for inclusive hosting.

\section{Project design and sample}

In October 2019, we listed 3,414 micro-entrepreneurs in areas where refugees operate businesses, from which we selected our main sample (inexperienced Ugandan business owners) and mentors

(experienced refugee and Ugandan business owners). The inexperienced Ugandan businesses were randomly assigned to a refugee mentor, a Ugandan mentor, a cash grant, information, cash and information, or control. Mentors were selected in order to have balance on refugee and Ugandan mentee characteristics (profit, years of experience, location) and match business owners in the same industry-gender cell.

Target population Our sample consists of 1,558 Ugandan tailors and salons with less than 5 years of industry experience and fewer than 3 employees. We chose these industries to meet the following criteria: microentrepreneurs would (1) be able to participate in mentorship, (2) have a skill that could be learned in mentorship, (3) be stable enough in to permit a high follow-up rate (4) work in areas where refugee mentors are available within $1-1.5 \mathrm{~km}$. We are also surveying refugees and Ugandan mentors to gather data on their business trends and perceptions. These data will 
be used to look at heterogeneous effects of mentorship by mentor characteristics, and permit a quasi-experimental test of the effects of mentorship on the mentor.

\section{$2.1 \quad$ Interventions}

We implemented five treatments in partnership with Young African Refugees for Integral Development (YARID), a Kampala-based NGO.

Treatment 1: Canvassing This treatment arm gives respondents "light touch" information about the existing aid-sharing framework in Uganda, together with information about refugee experiences. The YARID staff member provides information about the typical refugees experience, YARID's work in Uganda, and the aid-sharing policy, and takes the participant through an empathetic listening exercise (Kalla and Broockman, 2020).

Treatment 2: Grant This intervention offers a business grant worth 500,000 Ugandan Shillings (136 USD) without any information about or association with refugees. In a subsequent meeting, the YARID staff member accompanies the business owner to a shop, where the business owner must spent at minimum 300,000 UGX out of the 500,000 UGX grant on in-kind business-purpose purchases such as inventory, machinery, or rent payments for the business location. The remaining value of the grant is disbursed via mobile money.

Treatment 3: Grant and Canvassing This treatment provides a "heavy touch" that combines the information of Treatment 1 with the business grant of Treatment 2 .

Treatment 4: Refugee mentorship This treatment pairs inexperienced Ugandan business owners with experienced refugee business owners for a series of partially-structured, businessoriented mentorship meetings. The participant is informed that the business owner is in the same industry and of the same gender and may or may not be of the owner's same nationality. A YARID staff member arranges the meetings with the mentor and accompanies the mentee to the mentor's business. The YARID staff member is present for the conversations to assist with translation, prompt the initial conversation, and take notes. The YARID staff member can suggest topics of conversation such as skills and training that the mentee is interested in, customer service and retention strategies, finance, and management. The YARID staff member attends up to 6 meetings with the pairs, and encourages the mentees and mentors to continue meeting afterward.

Treatment 5: Ugandan mentorship This treatment is structured identically to Treatment 4, but pairs inexperienced Ugandan business owners with experienced Ugandan business owners.

Control Group This group receives no intervention and was not contacted by YARID. 


\subsubsection{COVID-19 Interruption}

The interventions were launched in late January of 2020 and suspended on March 20, 2020 due to COVID-19. At the time of the suspension, YARID had visited:

- $82 \%$ of the canvassing only treatment arm

- $75 \%$ of the grant and grant and canvassing arms for the first meeting to explain the program

- $33 \%$ for the second meeting to disburse the grant

- $83 \%$ of the mentorship treatment arms. $70 \%$ of the mentorship pairs met at least once, with $23 \%$ of those having met all six times

Businesses were prohibited from operating between the end of March and the middle of July. Before restarting the interventions, we are completing a midline survey in October 2020 to assess business survival, attrition, and the changes in outcomes since baseline given the large shock. From the midline, we will measure the treatment on the treated (ToT) effects - the effects after roughly six months among those who were reached by YARID before the suspension 11 The ToT effects will likely be under-powered due to the small number of businesses which will have been treated at that time.

The effect sizes and attrition at midline will be used to update power calculations and inform the design of interventions before we restart. If substantial portions of the sample cannot be reached, then treatment groups may be modified so that the restarted experiment is sufficiently powered for the main hypotheses (e.g., re-allocating some of the sample to grant + canvassing so that this group is sufficiently powered). This parallels the idea of adaptive field experiments, where the treatment probabilities change based on treatment effects from the midline. While not the original design of the study, the COVID-19 interruption allows for mid-treatment analysis of effect sizes and adaptation if necessary. The initial design and full midline effects will be reported in the paper even if interventions are modified for post-COVID implementation.

\section{$2.2 \quad$ Hypotheses and Outcomes}

\subsubsection{Primary Hypotheses and Outcomes: Hosting Policy and Profits}

We have four main hypotheses. They cover two main treatment arms - grants with canvassing (T3) and mentorship by a refugee (T4) - and relate to two main outcomes: summary indices of support for inclusive hosting and business outcomes. Specifically, we hypothesize that:

1. Grants with canvassing (T3) will increase support for inclusive hosting

2. Refugee mentorship (T4) will increase support for inclusive hosting

\footnotetext{
${ }^{1}$ The treatment on the treated specification will use the main ANCOVA specification below, except with indicators for receiving each treatment before the suspension, which will be instrumented with indicators for assignment to each treatment group.
} 
3. Grants with canvassing (T3) will increase business profits

4. Refugee mentorship (T3) will increase business profits

These outcomes will be measured as follows. Unless otherwise specified, each question is measured with a 5-point Likert scale and an option for "don’t know."

\section{Domain 1: Support for inclusive refugee hosting.}

- Overall, during coronavirus, I am in favor of Uganda hosting and assisting refugees.

- In July refugees from Congo were allowed to come to Uganda. They were tested for coronavirus, quarantined, and settled into camps. I am in favor of allowing refugees who test negative to move to Uganda right now.

- After coronavirus, I am in favor of Uganda hosting and assisting refugees.

- After coronavirus ends, Uganda should accept more refugees.

- During coronavirus, Uganda should relocate all refugees to live in the settlements, including those currently living in Kampala.

- For those who answered "agree" or "strongly agree": Should the relocation be permanent or only during coronavirus?

- Uganda should continue allowing refugees who already live in Uganda to work outside the settlements, according to any lockdown rules, during coronavirus.

- After coronavirus ends, Uganda should continue allowing refugees to work outside the settlements.

In domain 1, we hypothesize that T3 and T4 will increase support for inclusive hosting. We hypothesize $\mathrm{T} 1$ will also increase support but by less than T3 and T4.

\section{Domain 2: Business profits}

- What were the profits of your business during the last 30 days?

We will compute this measure as the answer to the above question minus the additional value of unpaid family labor used for the business (additional means relative to the control group mean). We will compute this value as the product of total unpaid hours spent on the business by the owner and family members (minus the control group average) times the marginal value of time. We will compute the marginal value of time according to the following rule. After all endline data is collected, we will test whether there is a significant (at the $5 \%$ level) difference in unpaid family hours across treatment groups, using Westfall-Young stepdown adjusted p-values to control for the

\footnotetext{
${ }^{2}$ We analyze the inverse hyperbolic sine transformed variable. If the business has closed, profits are included in the index as 0 .
} 
family-wise error rate. If there is a significant difference between any two treatment groups, and if the maximum significant difference is greater than $10 \%$ of unpaid labor hours in the control group, we will estimate the value of time by eliciting reservation wages from a random subset of the control group using a Becker-DeGroot-Marshak auction. Otherwise, we will estimate the marginal value of time using the average wages paid to employees in our sample. For intermediate surveys, we will rely on the latter method.

In domain 2, we hypothesize that grants (T2 and T3) and mentorship (T4 and T5) will improve business outcomes. We hypothesize that the effects of grants will be larger than the effects of mentorship, and the effects of mentorship by a Ugandan will not statistically differ from mentorship by a refugee. Finally, we hypothesize that mentorship will have a higher treatment effect for women than men, and grants will have a higher treatment effect for men than women.

Since our interventions have different associated costs per participant, we will quantify the amount per intervention relative to the increase in income and profit. We calculate the cost effectiveness by dividing the treatment's average effect size over the average intervention cost. For shifting policy preferences towards accommodation and inclusion of refugees, we hypothesize that canvassing (T1) will be the most cost effective, followed by the grant with canvassing (T3), and finally mentorship by a refugee (T4). For increasing business profits, we hypothesize that grants will be more costeffective than mentorship. Our findings will allow policymakers to use their relative weights on improving business outcomes and social cohesion to decide between the interventions if rankings of cost effectiveness differ across the two objectives.

\subsubsection{Secondary Outcomes and Hypotheses: Attitudes towards Refugees}

Our main interest in policy preferences towards refugees is likely correlated with - and potentially influenced by - the domains below. Overall, we expect canvassing (T1) and canvassing with a grant (T3) to affect policy preferences through changes in beliefs about the economic effects of refugees. We expect mentorship by a refugee (T4) to instead affect policy preferences through changes in social attitudes and induce additional social and business interactions with refugees.

\section{Domain 3: Knowledge of refugees and hosting policy}

- How many refugees in Uganda live outside of camps or settlements: all, most, some, few, or none? ("Some" or "few" will be considered correct answers)

- Are refugees allowed to live outside of the camps or settlements? ("yes" is correct)

- Are any of the international donations to refugees in Uganda shared with Ugandans? ("yes" is correct)

The outcomes will be dummy variables for correct answers. "Don't know" will be coded as incorrect. We hypothesize that the two treatments with information (T1 and T3) and contact with a 
refugee through mentorship (T4) will lead to higher levels of knowledge.

\section{Domain 4: Beliefs about economic effects of refugees}

- How do the [sector] businesses managed by people from other countries affect your business overall? Do they help you a lot, help you a little, hurt you a little, hurt you a lot, or have no effect on you? We will analyze the difference relative to the question "How do the [sector] businesses managed by Ugandans from your tribe affect your business overall?" to control for overall perceptions of competition. This difference will be measured using a dummy variable equal to 1 when the answer to the former question is weakly more positive than the answer to the latter question.

- Taking everything into consideration, would you say the overall economic effect of refugees on Uganda has been positive, negative, or neutral?

- How about the overall economic effect of refugees on you personally?

- How many refugees have skills and contribute to the economy?

\section{Domain 4a: Beliefs about economic effects of Congolese refugees}

- Taking everything into consideration, would you say the overall economic effect of Congolese on Uganda has been positive, negative, or neutral?

- How have access and quality of schools and health facilities been affected by Congolese in Kampala?

- How have rents been affected by Congolese in Kampala?

- How have prices of goods you buy, other than rents, been affected by Congolese in Kampala?

\section{Domain 4b: Beliefs about economic effects of Somali refugees}

- (These will be the same questions as above for Somalis.)

We hypothesize that T3 and T4 will shift beliefs towards refugees having a positive economic effect.

\section{Domain 5a: Beliefs that refugees receive too much aid}

- How many refugees have a lot of money? All, most, some, few, or none?

- How many refugees get more assistance than they need?

\section{Domain 5b: Beliefs that refugees can support themselves}

- How many refugees are able to support themselves financially without assistance? 
We hypothesize that T4 (mentorship by a refugee) will increase beliefs about ability of refugees to support themselves financially. We hypothesize that T1 and T3 will decrease beliefs that refugees receive too much aid.

\section{Domain 6: Social attitudes about refugees}

- What effect have refugees had on culture in Uganda? (Very positive, somewhat positive, neutral, somewhat negative, negative, or don't know)

- Social distance (measured on 5-point Likert scale; each item is treated as an independent component when calculating the index):

- I would be comfortable marrying a refugee.

- I would be comfortable having a refugee marry a member of my family.

- I would be comfortable having a refugee as a close, personal friend.

- I would be comfortable having a refugee as a neighbor.

- How many refugees deserve sympathy and support?

- A real donation the respondent chooses to allocate between refugee-focused charity program, a Ugandan-focused charity program, and themselves). We measure the amount donated to the refugee-focused program, controlling for the amount kept for themselves. This outcome will also be used to address concerns about experimenter demand effects.

- How safe do you feel walking around areas in Kampala where people from other countries live? You can say very safe, somewhat safe, neutral, somewhat unsafe, very unsafe, or that it depends on the nationality. (For the "depends on nationality" option, we will code as unsafe if any of the main refugee nationalities are listed). This variable will be differenced from the answer to the question "How safe do you feel walking around most areas in Kampala?" to isolate the comfort levels around foreigners specifically.

- Is there tension between Ugandans and people from other nationalities?

\section{Domain 6a: Social attitudes about Congolese refugees}

- Social distance (defined above) from Congolese

- What effect have Congolese had on culture in Uganda?

- Please tell us how the dress code has been affected by Congolese in Kampala. You can answer positive, negative, or no effect.

- How have acceptable behaviors (such as how people talk to each other) been affected by Congolese in Kampala?

\section{Domain 6b: Social attitudes about Somali refugees}


- These are the same questions as above asked about Somalis instead of Congolese.

We hypothesize that T4 will lead to more positive attitudes towards refugees.

\section{Domain 7: Contact with refugees by choice}

- How many of your business collaborators are from another country?

- Would you be open to collaborating with business owners from another country?

- In the last 30 days, have you bought supplies (such as materials for your business), tools, or machines from someone from another country?

- Have you ever had an apprentice or person from outside your household at your business who was learning skills but not paid who was from another country?

- Are any of your employees from a different country than you?

- In the past 30 days, how many people from another country have you contacted for any social reason, such as having a long conversation?

- Number of people from another country listed in the networks module. Networks are elicited from the question "Think of people who you would ask for advice or consider partnering with. Who are the 4 people you talk to most?".

We expect T4 (mentorship by a refugee) to induce additional business and social interactions captured in this domain.

\section{Domain 8: Contact with refugees by circumstance}

- How many people from other countries live in your neighborhood? Many, some, few, or none?

- How many businesses in your sector in this area are managed by people from another country?

- How many of your customers are from another country?

While these types of contact potentially shape baseline attitudes, we do not expect them to be affected by the treatments.

\subsubsection{Secondary Outcomes and Hypotheses: Economic Outcomes}

The goal of the project is to understand how attitudes co-evolve with economic outcomes. Humanitarian organizations often address these objectives separately, and our goal is to investigate whether these goals can be combined into integrated programs that accomplish both simultaneously. We therefore investigate the economic outcomes of the interventions, the potential mechanisms, and the potential wider effects on household well-being.

\section{Domain 9: Business practices}


- Is your business officially registered?

- Business capital. We sum two variables ${ }^{3}$

- If you were to sell all the business-related equipment you own right now, how much do you think you could make?

- If you were to sell all the inventory you own right now, how much do you think you could make?

- Over the past 7 days, how many hours did you work at this business?

- In the past year, how many times did you take out a loan for your business?

- How much total business-related debt do you currently have?

- Number of contacts listed in the networks module. Networks are elicited from the question "Think of people who you would ask for advice or consider partnering with. Who are the 4 people you talk to most?".

- Over the past year, how often did you spend money advertising your business? Every day, every week, every month, a couple times, or never?

- How often did you keep written books/accounting records? Always, frequently, sometimes, occasionally, or never?

- How often did you sell goods or provide services to customers on credit? For all sales, most sales, some sales, a few sales, or never?

- How often did you buy materials, tools, or machines for your business on credit? For all sales, most sales, some sales, a few sales, or never?

We hypothesize that grants (T2 and T3) will increase capital, while mentorship (T4 and T5) will increase number of contacts and practices like advertising and sales on credit.

\section{Domain 10: Household Well-Being}

- Total household income. This will be computed as the sum of 4 measures of income.

- What were the profits of your business during the last 30 days?

- What were the profits of [any other household-owned] businesses (excluding this one) during the last 30 days?

- How much wage income did you earn in the last 30 days?

- How much wage income did [other members of your household] earn in the last 30 days?

\footnotetext{
${ }^{3}$ We analyze the inverse hyperbolic sine transformation of the total.
} 
- Business survival, measured using an indicator for whether the main business is operating at the time of the survey

- How much money was your household able to save in the past 30 days?

- Compared to the average Ugandan in your neighborhood, how would you describe the economic situation of your household? Much better, somewhat better, about the same, somewhat worse, or much worse?

- Over the past 30 days, how often have you or anyone in your household gone without enough food to eat?

- Over the past 30 days, how often have you or anyone in your household struggled to afford basic household expenses (such as medicine, rent, school fees)?

- In the past 30 days, have you or anyone in your household had to sell assets (jewelry, furniture, clothing, tools, machines, land) in order to afford basic household expenses?

- In the past 30 days, has your household had to stop education for a child due to lack of finances?

We hypothesize that grants (T2 and T3) and mentorship (T4 and T5) will increase total household income. We hypothesize these treatments will have positive effects on household well-being, though we are likely under-powered to detect effects on specific outcomes.

\subsubsection{Secondary Outcomes and Hypotheses: Spillovers on Attitudes towards Other Tribes, Immigrants, and Gender}

The main goal of the project is to measure whether the interventions change preferences towards

refugees. If we find evidence for the main effects, we are also interested in how shifting attitudes towards one group or business outcomes co-evolve with other attitudes. Similarly, attitudes towards out-groups may change with business outcomes, independent of the messaging around refugees.

\section{Domain 11: Policy Preferences and Representation}

- Do you agree or disagree with the following statement: Uganda should accept more foreigners besides refugees.

- For foreigners, besides refugees, which option do you think Uganda should follow? (analyzed as 4 binary variables)

- Allow them to stay in Uganda as long as they want

- Allow them to stay in Uganda for a limited time

- Allow current residents to stay but stop new immigration 
- Restrict all new immigration to the country and force foreigners in country to leave Uganda

- How satisfied are you with the LC1 for this area?

- How satisfied are you with the MP for this area?

We will not compute an index measure within this domain. If the main hypothesis holds - that T1, T3, and T4 increase support for inclusive refugee hosting policies - we hypothesize that they will increase support for non-refugee immigrants. We additionally hypothesize that respondents will attribute some credit for all treatments, or their slightly improved well-being overall, to their LC1 and MP representatives and offer increased support.

\section{Domain 12: Beliefs about economic effects of non-refugee immigrants}

- Taking everything into consideration, would you say the overall economic effect of foreigners other than refugees on Uganda has been positive, negative, or neutral?

- How about the overall economic effect of foreigners other than refugees on you personally?

\section{Domain 13: Social attitudes about other non-refugee immigrants}

- What effect have foreigners besides refugees had on culture in Uganda?

We hypothesize that beliefs and attitudes about non-refugee immigrants will shift in the same direction as beliefs and attitudes about refugees: T1, T3, and T4 will shift beliefs about economic effects of non-refugee immigrants, while only T4 (mentorship by a refugee) will shift social attitudes towards non-refugee immigrants.

\section{Domain 14: Contact with Ugandans from another tribe}

- How many of your customers are Ugandans from a different tribe?

- How many businesses in your sector in this area are managed by Ugandans from another tribe?

- How many of your business collaborators are Ugandans from a different tribe?

- Would you be open to collaborating with Ugandans from other tribes?

- Have you ever had an apprentice or person from outside your household at your business who was learning skills but not paid who was from another tribe?

- Are any of your employees from a different tribe than you?

- In the past 30 days, how many people from a different tribe have you contacted for any social reason, such as having a long conversation? 
- Number of people from another tribe listed in the networks module. Networks are elicited from the question "Think of people who you would ask for advice or consider partnering with. Who are the 4 people you talk to most?".

\section{Domain 15: Beliefs about economic effects of Ugandans from another tribe}

- How do the [sector] businesses managed by Ugandans from a different tribe affect your business overall? Do they help you a lot, help you a little, hurt you a little, hurt you a lot, or have no effect on you? This will be the difference from the question "How do the [sector] businesses managed by Ugandans from your tribe affect your business overall?" to control for overall perceptions of competition.

\section{Domain 16: Social attitudes about Ugandans from another tribe}

- Social distance (defined above) from Ugandans from a different tribe

We do not anticipate the treatments will affect attitudes towards or contact with other tribes.

\section{Domain 17: Gender roles}

- Do you share all of your profits from this business with your spouse?

- Who decides how the household's money is spent?

If female entrepreneurs realize an increase in business profits (through grants and mentorship), we hypothesize that they will have increased influence over their income and household income.

The domains above were asked during the baseline, and we intend to ask them during an inperson endline. However, the midline survey is an abbreviated version conducted over the phone due to COVID-19, and the endlines may need also need to consist of a subset of the above questions.

\section{Statistical analysis}

We will measure intent-to-treat effects using the following ANCOVA specification:

$$
y_{i t}=\sum_{j=1}^{5} \beta_{j} T_{j i}+\gamma y_{i 0}+\delta M_{i 0}+\eta X_{i}+\theta_{t}+\alpha_{i}+\epsilon_{i t} .
$$

$y_{i t}$ is an outcome for individual $i$ measured at time $t$ with $t=0$ corresponding to baseline (pretreatment) values, $M_{i 0}$ is an indicator for a missing value of $y_{i 0}, T_{j i}$ are treatment assignment dummies for treatment groups $j=\{1,2,3,4,5\}, X_{i}$ is a vector of baseline controls chosen through double lasso, $\theta_{t}$ is a survey round fixed effect, $\alpha_{i}$ is a strata fixed effect, and $\epsilon_{i t}$ is an error term 4

\footnotetext{
${ }^{4}$ In addition to the individual treatment arms, we will report the tests for $\mathrm{T} 1+\mathrm{T} 3$ (any information), T2 $+\mathrm{T} 3$ (any grant), and T4 + T5 (any mentorship).
} 
Standard errors will be clustered at the individual level. We will run separate lassos for each dependent variable and include all possible controls from the baseline in each.

We will also report treatment-on-the-treated estimates measured in October 2020, before all interventions are completed. We will estimate the marginal effect of the number of mentorship meetings by including the number of meetings held (between 0 and 6) in (1). We will estimate the marginal effect of receiving the grant in cash (as opposed to in-kind) by including the percentage of the grant taken in cash in (1).

\subsection{Measurement}

Indices will be constructed as in Anderson (2008). Index components with 95\% or more of respondents providing the same answer will be dropped.

We will transform variables in the following ways:

- Likert scales and other categorical variables will be transformed into binary measures (i.e. "very positive" and "somewhat positive" =1). Neutrals will be resolved towards the smaller group in order to maximize statistical power; we will report if changing this assignment mechanism changes results. "Don't know" and other missing values will not be included in the index; we will not impute missing values for outcome variables. We will impute missing values for control variables using the mean.

- Monetary measures including profit, revenue, capital, inventory, debt, \& savings:

- Values will be winsorized at the 1st and 99th percentiles within each survey round.

- Nominal values will be converted to real values using the CPI from the Uganda Bureau of Statistics.

- If the respondent declines to provide an exact amount but provides a range (pre-specified on the survey), we will use the midpoint of the range. For firms in the top range, the median of firms in the top range with reported point estimates will be used.

- Values will be transformed using the inverse hyperbolic sine. We will also report treatment effects on 1) levels and 2) quantile-transformed outcomes as in Delius and Sterck (2020) 5

- For firms that close down, values will be recorded as 0 , and additional analysis will be conducted conditional on surviving.

\footnotetext{
${ }^{5}$ We will use the quantiles in the control group and then interpolate the quantile values for the treatment group based on the control group quantiles. Values exceeding the maximum (minimum) in the control group will interpolated using a linear interpolation defined by OLS on values in the 95th through 100th (0th through 5th) percentiles.
} 


\section{$3.2 \quad$ Heterogeneity}

For main hypotheses 1 and 2, we will explore heterogeneity by:

- Gender: we hypothesize that effects on policy preferences will not be statistically different by gender

- Refugee status and nationality of YARID canvasser and mentor: we hypothesize that Congolese canvassers and mentors (the only group large enough to meaningfully isolate) will have larger effects on outcomes specifically related to Congolese.

- Baseline business profit (created at stratification): we hypothesize that effects will be larger for less profitable businesses at baseline.

- Baseline support for inclusive hosting (created at stratification): we hypothesize that effects will be larger among those with less initial support for inclusion.

- Baseline beliefs about economic effects of refugees (index): we hypothesize that effects will be larger among those with more negative baseline beliefs about refugees' economic effects.

- Baseline social attitudes about refugees (index): we hypothesize that effects will be larger among those with less baseline social tolerance of refugees.

- Baseline contact with refugees by choice (index): we hypothesize that effects will be larger among those with less baseline contact with refugees.

- Baseline contact with refugees by circumstance (index): we hypothesize that effects will be larger among those with less baseline contact with refugees.

- Baseline knowledge of whether international donations to refugees are shared with Ugandans: we hypothesize that effects will be larger among those with less baseline knowledge of the policies.

- Baseline profits of the mentor: we hypothesize that effects will be larger among those with more profitable mentors

- Midline worry about COVID-19: we hypothesize that effects will be larger among those who are more worried about COVID-19

For main hypotheses 3 and 4, we will explore heterogeneity by:

- Gender: we hypothesize that effects of cash will be larger for men and the effects of mentorship will be larger for women

- Baseline business practices: we hypothesize that effects will be larger among those with fewer baseline standard practices

- Baseline business networks size (number of businesses listed in networks module): we hypothesize that effects will be larger among those with fewer social contacts at baseline. 
- Baseline characteristics of the mentor

- Profit: we hypothesize that effects will be larger among those with more profitable mentors.

- Experience: we hypothesize that effects will be larger among those with more experienced mentors.

- Driving distance from mentee: we hypothesize that effects will be larger among those with mentees who live closer.

Heterogeneous treatment effects will be estimated by including an interaction of the heterogeneity and the treatment status in the main ANCOVA specification.

\subsection{Multiple measurement}

We plan to have one midline (October 2020) and at least two endline surveys. At the midline, only part of the sample will have been offered the treatment, due to the interruption of COVID-19, and outcomes will be measured 7-9 months after the treatment. Following the midline, the treatments will resume, and endlines are planned for approximately 1 and 3 months after the completion of all interventions. If the effects attenuate over time, pooling these survey waves may not capture the short-term effects. We will therefore run additional specifications that allow for time-varying effects with separate coefficients for the number of months since the treatment. We will plot the treatment effects over time for the main outcome variables and evaluate whether an interaction with each treatment of "treated within X months" (pooling the under-powered monthly effects) captures a short-term effect.

\subsection{Multiple hypotheses}

First, we will report all of the outcomes listed here that are asked in the survey. For the four primary hypotheses, we will use Westfall-Young stepdown adjusted p-values to control for the family-wise error rate. Within each domain, we will follow Anderson (2008) to create indices to reduce measurement error and the number of tests, and use sharpened q-values for individual survey questions within domains to control for the false discovery rate. When using alternative variable transformation (see Section 3.1) we will treat these estimates as distinct domains.

\subsection{Attrition}

We will test for attrition using the main specification (omitting $X \mathrm{~s}$ and baseline measures) with an indicator for whether a survey was completed as the dependent variable. We will test whether attrition is related to any treatment status (the joint hypothesis that treatment coefficients equal 0 ) and individual treatments. If any test for attrition is significant at the $5 \%$ level, we will estimate Lee bounds (Lee, 2009). 


\section{COVID-19 Hypotheses and Tests}

Given the significant shock that occured in the middle of the study, we will also test two additional hypotheses from the panel data. We hypothesize that refugees face higher barriers to economic recovery due to differences in access to capital, government assistance measures, discrimination, and social networks. This hypothesis therefore covers the outcomes in Domain 2, and some of the household welfare measures in domain 10. In addition, we ask the following questions in the midline to address this hypothesis:

\section{Domain 18: COVID-19 Household Shock}

- In total, about how much income did your family earn during the 4 months of the lockdown (April - July)? Do not count money that you borrowed.

- During the lockdown, how often did you or anyone in your household go without enough food to eat? Always, often, sometimes, or never?

- During the lockdown, how often did you or anyone in your household struggle to afford basic household expenses other than food (such as medicine, rent, school fees)?

- During the lockdown, did you or anyone in your household have to sell assets (jewelry, furniture, clothing, tools, machines, land) in order to afford basic household expenses?

- How much did you borrow during the lockdown to pay for basic necessities like food, housing, and medicine?

To evaluate the additional hypotheses around COVID-19, we will run a difference-in-differences specification across time and refugee status, weighting Ugandans by their probability of being selected in the listing in accordance with their experience. Treatments distributed before the COVID-19 suspension will be included as control variables, along with baseline controls selected by a lasso.

Finally, we also hypothesize that the fear of the virus, combined with the negative economic shock, will lead to an increase in xenophobia and opposition to the inclusive hosting policies. We hypoth-

esize that Ugandans who are more worried about contracting COVID-19 and those who faced a more severe economic shock will decrease their support for inclusive hosting relatively more between baseline and midline.

\section{References}

Anderson, Michael L., "Multiple inference and gender differences in the effects of early intervention: A reevaluation of the Abecedarian, Perry Preschool, and Early Training Projects," Journal of the American Statistical Association, 2008, 103 (484), 1481-1495. 
Benhassine, Najy, David McKenzie, Victor Pouliquen, and Massimiliano Santini, "Impact Evaluation of the Entreprenant Status in Cotonou," AEA RCT Registry, 2015.

Delius, Antonia and Olivier Sterck, "Cash Transfers and Micro-Enterprise Performance: Theory and Quasi-Experimental Evidence from Kenya," Working Paper, 2020.

Kalla, Joshua and David Broockman, "Reducing exclusionary attitudes through interpersonal conversation: evidence from three field experiments," American Political Science Review, 2020, 114 (2), 410-425.

Lee, David S, "Training, wages, and sample selection: Estimating sharp bounds on treatment effects," The Review of Economic Studies, 2009, 76 (3), 1071-1102. 